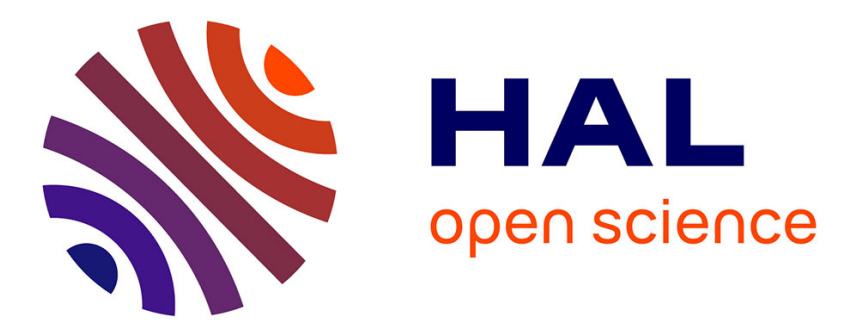

\title{
France-algérie pour un traitement commun du passé de la guerre d'indépendance
}

Sylvie Thénault

\section{To cite this version:}

Sylvie Thénault. France-algérie pour un traitement commun du passé de la guerre d'indépendance.

Vingtième siècle. Revue d'histoire, 2005, 85 (1), pp.119. 10.3917/ving.085.0119 . hal-02355826

\section{HAL Id: hal-02355826 \\ https://hal.science/hal-02355826}

Submitted on 12 Nov 2019

HAL is a multi-disciplinary open access archive for the deposit and dissemination of scientific research documents, whether they are published or not. The documents may come from teaching and research institutions in France or abroad, or from public or private research centers.
L'archive ouverte pluridisciplinaire HAL, est destinée au dépôt et à la diffusion de documents scientifiques de niveau recherche, publiés ou non, émanant des établissements d'enseignement et de recherche français ou étrangers, des laboratoires publics ou privés. 


\title{
FRANCE-ALGÉRIE POUR UN TRAITEMENT COMMUN DU PASSÉ DE LA GUERRE D'INDÉPENDANCE
}

\author{
Sylvie Thénault
}

Presses de Sciences Po | «Vingtième Siècle. Revue d'histoire »

2005/1 n ${ }^{\circ} 85 \mid$ pages 119 à 128

ISSN 0294-1759

ISBN 2724630025

Article disponible en ligne à l'adresse :

https://www.cairn.info/revue-vingtieme-siecle-revue-d-histoire-2005-1-page-119.htm

Distribution électronique Cairn.info pour Presses de Sciences Po.

(C) Presses de Sciences Po. Tous droits réservés pour tous pays.

La reproduction ou représentation de cet article, notamment par photocopie, n'est autorisée que dans les limites des conditions générales d'utilisation du site ou, le cas échéant, des conditions générales de la licence souscrite par votre établissement. Toute autre reproduction ou représentation, en tout ou partie, sous quelque forme et de quelque manière que ce soit, est interdite sauf accord préalable et écrit de l'éditeur, en dehors des cas prévus par la législation en vigueur en France. Il est précisé que son stockage dans une base de données est également interdit. 


\title{
POINT DE VUE
}

\section{FRANCE-ALGÉRIE \\ POUR UN TRAITEMENT COMMUN \\ DU PASSÉ DE LA GUERRE \\ D'INDÉPENDANCE}

\author{
Sylvie Thénault
}

Devant les polémiques toujours vivaces de part et d'autre de la Méditerranée et l'absence de gestion commune entre la France et l'Algérie de la mémoire de la guerre d'indépendance algérienne, les modalités du rapprochement franco-allemand offrentelles la voie à suivre ? Pour Sylvie Thénault, rien n'est moins sûr tant les divergences sont notables, notamment parce que la France et l'Algérie vivent sous des régimes politiques différents, qu'elles sont toujours liées par des rapports d'inégalité et que le conflit qui les a opposées a été marqué par le brouillage de la frontière entre victimes et bourreaux. La gestion franco-algérienne de leur passé commun semble en conséquence un objectif qui reste à atteindre.

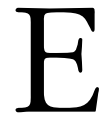

n France, la "guerre d'Algérie " est régulièrement dénoncée comme un événement nié, oublié, occulté, un événement tabou que l'État aurait cherché à enfouir sous une lourde chape de plomb, impression renforcée par le silence des anciens combattants à leur retour. Les polémiques, qui se sont succédé depuis trois ans pour dénoncer la torture ou le massacre des harkis, ont très largement gagné l'espace public ${ }^{1}$. À son tour décrite par les métaphores nées de la dénonciation du régime de Vichy, " heures sombres ", "pages noires " de l'histoire de la France contemporaine, la guerre d'indépendance algérienne a pris le relais de la seconde guerre mondiale comme séquence historique culpabilisante pour les Français. La gestion du passé vichyste, avec ses débats, ses procès, ses plaques commémoratives, ses discours, ses tentatives de réparation... sert d'ailleurs de référence et de modèle pour réclamer une reconnaissance officielle du conflit franco-algérien et de ses victimes ${ }^{2}$. Elle fonde ainsi l'argumentation des défenseurs du devoir de mémoire : pourquoi la France ne mettrait-elle pas en ouvre, pour

1. Tramor Quemeneur a retracé les étapes des polémiques sur la torture : "Le débat sur les tortures dans la guerre d'Algérie, juin 2000-septembre 2001 ", Regards sur l'actualité, 276, décembre 2001, p. 29-41. À l'automne 2003, la sortie du livre de Georges-Marc Benamou s'est accompagnée d'une opération médiatique et commerciale fracassante, ainsi que du dépôt d'une plainte visant Pierre Messmer, ancien ministre des Armées, pour crime contre l'humanité dans l'" abandon " des harkis : Un mensonge français. Retours sur la guerre d'Algérie, Paris, Robert Laffont, 2003.

2. Sur la gestion du passé du régime de Vichy, cf. Éric Conan et Henry Rousso, Vichy, un passé qui ne passe pas, Paris, Gallimard, coll. "Folio Histoire ", 1996 (rééd.). Sur les liens entre la gestion de ce passé et celui de la guerre d'indépendance algérienne, cf. Henry Rousso " La guerre d'Algérie, la mémoire et Vichy ", L'Histoire, 266, juin 2002, p. 2829. Cet article a bénéficié de ses précieux conseils. Qu'il en soit remercié, ainsi qu'Anne-Marie Pathé pour sa relecture. 
la guerre d'indépendance algérienne comme pour la seconde guerre mondiale, une politique de reconnaissance ou de réparation, voire les deux à la fois?

Du côté algérien, la "guerre de libération ", aussi appelée "Révolution ", a constitué un corpus de références historiques à l'appui du pouvoir politique. Au contraire de l'enfouissement, c'est une mémoire manipulée de l'événement qui a été forgée et maniée par le pouvoir ${ }^{1}$. Les dirigeants algériens ont érigé la figure du moudjahid le combattant - en héros, attribué à l'Armée de libération nationale (ALN), reconvertie en Armée nationale populaire après 1962, les mérites de la victoire; ils ont puisé dans leur participation à la lutte pour l'indépendance une justification à leur accès aux plus hautes fonctions de l'État, maintenant le pays dans une véritable "culture de guerre ${ }^{2}$.

D'emblée, les usages de ce passé s'annoncent discordants de part et d'autre de la Méditerranée. La dénomination même du conflit atteste des divergences d'approche. En France, bien avant que la loi admette officiellement cette terminologie, elle a été désignée, dans le langage courant, comme la "guerre d'Algérie ", expression évoquant une campagne militaire ; "campagne d'Algérie ", cette guerre s'apparente à une opération de conquête, ou plutôt, une opération de reconquête après plus d'un siècle d'occupation. En Algérie, elle est l'acte de naissance de la nation algérienne : "Révolution ", elle a transformé les "Français musulmans ", sujets coloniaux, en Algériens collectivement souverains ; "guerre de libération ", elle a affranchi leur territoire de la tutelle française ${ }^{3}$.

1. Cf. Benjamin Stora, La gangrène et l'oubli. La mémoire de la guerre d'Algérie, Paris, La Découverte, 1992, ainsi que Gilles Manceron et Hassan Remaoun, D'une rive à l'autre. La guerre d'Algérie de la mémoire à l'bistoire, Paris, Syros, 1993.

2. L'expression est de Benjamin Stora, notamment dans $L a$ gangrène et l'oubli, op. cit. Il s'agit d'une culture glorifiant l'union des Algériens dans la mobilisation contre l'ennemi colonial et valorisant à l'excès la lutte armée.
En préface à la publication d'un colloque réunissant des historiens des deux rives de la Méditerranée, Charles-Robert Ageron constatait cet écart, tout en le relativisant, grâce à une comparaison avec le cas franco-allemand : "Parce que nous historiens français avons l'expérience des longs efforts qui furent nécessaires avant d'aboutir à un traitement impartial des guerres franco-allemandes, nous savons que l'heure n'est pas encore venue où l'on pourrait écrire une histoire de la guerre d'Algérie qui serait acceptée par tous les Algériens et par tous les Français ${ }^{4}$. " Le parallèle peut être transposé sur le terrain mémoriel : sur un tel socle de divergences, c'est une gestion nationale de ce passé, propice à la perpétuation des litiges, qui a été mise en ouvre par chacun des deux pays, en fonction de leur conjoncture politique et de leurs enjeux internes. Mais, cette guerre étant un moment d'histoire partagée par la France et l'Algérie, dans le prolongement de la longue période coloniale, quel sens a une gestion séparée de ce passé ?

\section{O UN PASSÉ AUX ENJEUX INTERNES PRIORITAIRES}

En France, le souvenir de la guerre d'indépendance algérienne est resté vif et souvent douloureux chez ceux qui l'ont vécue ou l'ont reçue en héritage de l'histoire familiale : anciens combattants, harkis, piedsnoirs, immigrés, militants politiques engagés contre la guerre ou pour les droits de l'Homme... Formées dans un premier temps pour représenter leurs intérêts, comme la FNACA (Fédération nationale des anciens combattants d'Algérie, Maroc, Tunisie) pour les anciens combattants, ces associations ont changé de nature dans les

3. L'expression "guerre d'indépendance algérienne", neutre par rapport aux enjeux franco-algériens, restitue mieux la nature de l'événement sur le plan historique : une guerre d'où est sortie la nation algérienne.

4. Dans sa préface à La guerre d'Algérie et les Algériens, Charles-Robert Ageron (dir.), Paris, IHTP/Armand Colin, 1997 , p. 4. 
années 1990 pour investir le terrain de la revendication mémorielle. Elles ont été à l'origine d'une abondante production sur l'histoire de cette guerre, par la publication de témoignages, l'organisation de réunions et manifestations publiques, la communication dans les médias, la diffusion de leur propre presse, tracts et affiches, voire par la réalisation de documentaires télévisés. Elles ont aussi, parfois, tenté de provoquer un procès en déposant une plainte pour obtenir un débat public, national, sur l'événement les préoccupant ${ }^{1}$. Les procès de la période vichyste, celui de Paul Touvier, ancien dirigeant de la Milice du Rhône, jugé en 1994, et celui de Maurice Papon, ancien secrétaire général de la préfecture de Bordeaux, jugé en 1998, ont créé un modèle de reconnaissance et de réparation que les associations défendant les victimes de la guerre d'indépendance algérienne tentent de reproduire.

La mémoire de cette guerre, en France, ne relève donc pas uniquement d'une gestion étatique. L'existence et l'activité de ces associations témoignent d'un dynamisme de la mémoire, indépendant de l'État, les anciens de la guerre ou leurs héritiers véhiculant leurs propres souvenirs et leurs propres interprétations de l'événement. La gestion même de ce passé par l'État, en France, s'inscrit dans un rapport de forces avec ces associations mues, dans les années 1990, par l'idée que se souvenir, reconnaître, réparer, est un devoir. Voyant en l'État le grand ordonnateur du silence

1. L'activité des deux associations se consacrant au 17 octobre 1961, jour de manifestation des Algériens à Paris, violemment réprimée, en est représentative. Le collectif antiraciste "Au nom de la mémoire ", formé en 1991, a ainsi publié un livre d'Anne Tristan, Le silence du fleuve, autoédité, et un documentaire du même nom. Il s'est aussi battu pour obtenir la pose d'une plaque commémorative à Paris, en 2001. L'association " 17 octobre contre l'oubli ", de son côté, a vainement porté ses efforts sur la qualification de cette répression comme crime contre l'humanité. L'ouvrage collectif, Le 17 octobre 1961, un crime d'État à Paris, sous la direction d'Olivier Le Cour Grandmaison, paru aux Éditions de La Dispute en 2001, est révélateur de cette démarche. Des associations de harkis, comme le "Collectif national justice pour les harkis et leurs familles " ou le "Comité harkis vérité ", ont déployé le même type d'activité. collectif autour de l'événement, ces militants l'ont suscité, provoqué et contraint d'agir. Pendant longtemps en effet, les pouvoirs publics ont brillé par leur volonté de taire ce passé. La voie judiciaire a été bloquée de différentes manières : aux amnisties s'est ajouté le caractère prescriptible des crimes de guerre, interdisant les poursuites de ce chef, et une définition du crime contre l'humanité évitant son application à la guerre d'indépendance algérienne $^{2}$. Et il a fallu attendre 1999 pour qu'une loi admette officiellement la terminologie de "guerre d'Algérie ${ }^{3}$ ".

Depuis cette date, en réponse aux pressions des associations défendant le devoir de mémoire, stimulées par le procès Papon, et pour calmer les différentes polémiques, les pouvoirs publics français ont multiplié les gestes de reconnaissance, inscrivant progressivement la guerre d'indépendance algérienne dans l'espace public et le calendrier national. Une plaque commémorant la mort des manifestants du 17 octobre 1961 a été posée au pont SaintMichel en octobre 2001, tandis qu'une journée nationale d'hommage aux harkis était décidée le 25 septembre de la même année. Récemment, le 5 décembre a été choisi comme "journée nationale d'hommage aux "morts pour la France" pendant la guerre d'Algérie et les combats du Maroc et de la Tunisie ${ }^{4}$ ". En réponse aux exigences d'une "vérité "sur l'événement, une circulaire de Lionel Jospin, souhaitant que "les chercheurs disposent d'un large accès aux archives publiques relatives à ces événements ", a prescrit diverses mesures pour le faciliter $^{5}$. Les responsables de l'Éducation nationale, de leur côté, ont multiplié les

2. Sur les amnisties, cf. Stéphane Gacon, L'amnistie, de la Commune à la guerre d'Algérie, Paris, Le Seuil, coll. "L’Univers historique ", 2002. Sur l'élaboration française du crime contre l'humanité, cf. Éric Conan et Henry Rousso, Vichy, un passé qui ne passe pas, op. cit., p. 183-200.

3. Loi n 99-882 du 18 octobre 1999, "relative à la substitution, à l'expression "aux opérations effectuées en Afrique du Nord", de l'expression "à la guerre d'Algérie ou aux combats en Tunisie et au Maroc" ".

4. Décret n²003-925 du 26 septembre 2003.

5. Circulaire du 13 avril 2001. 
journées de formation à destination des enseignants et suscité des publications à vocation pédagogique ${ }^{1}$. Choix de lieux, de textes et de dates commémoratives, accès aux archives, enseignement : les autorités politiques françaises ont entrepris d'utiliser les divers moyens en possession des pouvoirs publics pour intervenir sur le terrain mémoriel.

Du côté algérien, la gestion du passé a obéi à des modalités très différentes. Dans le cadre d'un régime non démocratique, où règne un parti unique puisant la source de sa légitimité dans la guerre, l'État a mobilisé les ressources en sa possession pour contrôler l'écriture du passé, sa connaissance et sa diffusion. À la source de la production historique, la mainmise sur la recherche est allée jusqu'au projet de fermer les centres d'histoire des universités pour n'en garder qu'un, à Alger, en 1984, projet qu'une forte opposition des universitaires a pu faire échouer ${ }^{2}$. Les programmes et manuels scolaires ont consacré une place prépondérante à la période coloniale et à la "guerre de libération ", l'insurrection du $1^{\text {er }}$ novembre 1954 tenant lieu de "mythe fondateur $^{3}$ " de la nation algérienne. Autour de cette date, l'approche commémorative et politique s'est substituée à l'histoire pour fonder dans l'espace public une représentation figée de l'événement : celle d'un peuple algérien uni, sans division, en armes, triomphant du colonisateur. Les publications, quant à elles, étaient monopolisées par un éditeur étatisé, la Société nationale d'édition et de diffusion (SNED).

Cet important contrôle, combiné à l'absence de pluralisme politique, a empêché l'émergence d'un débat public sur l'événe-

1. En août 2001, par exemple, l'Éducation nationale a organisé une université d'été : Apprendre et enseigner la guerre d'Algérie et le Maghreb contemporains, publié par le CRDP de l'académie de Versailles.

2. Cf. Gilles Manceron et Hassan Remaoun, D'une rive à l'autre. La guerre d'Algérie de la mémoire à l'histoire, op. cit., p. 57.

3. Cf. Hassan Remaoun, "Pratiques historiographiques et mythes de fondation. Le cas de la guerre de libération à travers les institutions algériennes d'éducation et de recherche ", in Charles-Robert Ageron (dir.), op. cit., p. 315. ment. L'année 1988 a donc marqué un tournant, les émeutes du mois d'octobre contraignant l'État algérien à accepter le pluralisme. Accompagnant ou révélant le changement, les publications d'anciens acteurs de la guerre se sont multipliées et les ouvrages publiés en France ont pu être diffusés en Algérie ${ }^{4}$. Parmi d'autres signes de cette évolution, Messali Hadj, dirigeant historique du mouvement national algérien avant 1954, a réintégré le panthéon national et l'assassinat d'Abbane Ramdane, dirigeant du Front de libération nationale (FLN) éliminé par ses pairs en 1957, a pu être discuté 5 .

Surtout, le droit de former des associations, reconnu en 1989, a permis la création de fondations, comme la Fondation du 8 mai 1945, la Fondation Mohammed Boudiaf ou la Fondation el-Mokrani. Agissant dans des domaines très divers, de l'humanitaire au financement de programmes de recherches, les fondations ont pu être considérées comme l'expression d'une société civile indépendante et bénéficier de subventions, notamment de l'Union européenne. L'histoire étant, par ailleurs, très utilisée dans la légitimation du pouvoir, elles ont aussi investi le terrain mémoriel et historique par l'organisation de colloques, débats et rencontres. Les universitaires ont pu y trouver des espaces de discussion et des moyens pour financer leurs activités. Même si, depuis 1992, avec l'interruption

4. C'est le cas des mémoires de Saad Dahlab : Pour l'indépendance de l'Algérie, mission accomplie, Alger, Dahlab, 1989, ou encore de celles d'Hocine Ait Ahmed : Mémoires d'un combattant, Paris, Messinger, 1983, réédité à Alger par Bouchène en 1990. Les volumes d'Yves Courrière, par exemple, ont été réédités par Rahma en Algérie, en 1992 : La guerre d'Algérie, 4 vol., Paris, Fayard, 1968-1971. Pour plus de précisions, cf. Gilles Manceron et Hassan Remaoun, op. cit., p. 260-263.

5. Après la réédition, en 1990, en Algérie, de la biographie écrite par Benjamin Stora (Messali Hadj (1898-1974), pionnier du nationalisme algérien, Paris, L'Harmattan, 1986), Messali Hadj a fait l'objet d'un colloque le 30 mars 1999. Cf. Benjamin Stora, " Maroc-Algérie. Retour du passé et écriture de l'histoire ", Vingtième Siècle. Revue d'bistoire, 68, octobredécembre 2000, p. 109-118. L'assassinat d'Abbane Ramdane a été discuté, notamment, à l'occasion de la parution de sa biographie écrite par Mameri Khalfa : Abbane Ramdane, béros de la guerre d'Algérie, Paris, L'Harmattan, 1988. 
du processus électoral et de la démocratisation en cours, ces fondations ont perdu de leur indépendance, la fenêtre d'opportunité ouverte par la démocratisation ne s'est pas refermée et les ouvrages relatifs à la guerre d'indépendance connaissent un grand succès ${ }^{1}$.

Ainsi, chronologiquement et dans ses modalités, la gestion du passé de la guerre d'indépendance algérienne a suivi des voies propres à chaque pays. En privilégiant les enjeux internes, la France et l'Algérie ont négligé la réflexion sur une gestion commune; elles ne l'ont même pas imaginé, même pas recherché. Pourquoi?

\section{O UNE GESTION COMMUNE IMPENSABLE}

La différence de régime entre les deux pays apporte un premier élément d'explication à cette absence. Elle a donné à la gestion politique de la mémoire des configurations divergentes, qui ne se prêtaient pas à une gestion commune. En France, les représentations collectives du passé recèlent des enjeux qui sont publiquement débattus, qui font l'objet de rapport de forces entre l'État et les acteurs sociaux, collectivement organisés, alors qu'en Algérie, l'État a tenté de confisquer la gestion du passé. La différence de régime impose donc des modalités différentes de relation au passé et de gestion de l'histoire dans le cadre national : en France, peuvent s'exprimer des revendications mémorielles et l'État doit composer avec elles, avec les campagnes médiatiques, avec l'action d'une justice indépendante, tandis que, en Algérie, le régime de parti unique allait de pair avec la volonté d'un contrôle des représentations du passé. En France, les résurgences de la guerre ont ainsi été liées au développement de campagnes d'opinion et d'un débat public, en réponse à une activité politique, citoyenne, alors

1. Réédité par Casbah-Éditions, le livre de Gilbert Meynier a ainsi été rapidement épuisé : Histoire intérieure du FLN, Paris, Fayard, 2002. qu'en Algérie, la chronologie de la gestion du passé a suivi celle de la démocratisation du régime. Il fallait que le régime admette le pluralisme pour que la période soit publiquement et collectivement revisitée. La réhabilitation des anciens dirigeants bannis, comme Messali Hadj, a d'ailleurs servi d'indicateur pour mesurer l'ouverture du régime à la démocratie.

La différence entre les régimes crée ainsi un obstacle à la gestion commune de ce passé, car chaque régime le gère avec son propre fonctionnement, selon ses propres impératifs. Or, adopter une gestion commune suppose que les deux régimes suivent des règles similaires ou, au moins, consensuelles, pour travailler ensemble. La question n'est pas ici de savoir si deux régimes différents, l'un démocratique et l'autre pas, peuvent collaborer, car gérer un passé commun va au-delà de l'alliance politique, de la coopération économique ou culturelle. Gérer un passé commun, c'est être dans l'échange, s'immiscer dans les affaires l'un de l'autre, accepter l'ingérence réciproque dans un domaine hautement sensible. Partager la gestion d'une histoire commune, c'est se conférer mutuellement une légitimité par une reconnaissance symétrique comme partenaire. La comparaison avec le rapprochement franco-allemand, ici, clarifie l'idée. Il s'agissait, en réalité, d'un rapprochement avec la RFA, régime frère, et non d'un rapprochement avec la RDA, régime inscrit dans le bloc ennemi, dans un contexte de guerre froide.

Le rapport ancien entretenu par la France et l'Algérie bloque aussi une gestion commune de ce passé. Ces deux pays n'ont pas été des États se déclarant la guerre, sur une question les opposant. La France colonisait l'Algérie, elle la dominait et cette dernière s'est construite, en tant que nation, en tant qu'État souverain, contre la France, dans un renversement de la domination coloniale, dans une émancipation de la tutelle française. L'Algérie se définit, de façon complexe, avec et contre 
la France : avec, car l'héritage de 132 ans de colonisation ne peut être balayé, il est irréversible; contre, car c'est bien de la lutte contre la colonisation qu'est née l'Algérie d'aujourd'hui. Dans la tension entre acceptation de l'héritage et son refus - car il est né du mal colonial -, le rapport à la France est problématique ${ }^{1}$. Du côté français, ce passé est source de culpabilité : non seulement des crimes ont été commis, pendant la période coloniale et pendant la guerre d'indépendance, mais en outre, la décolonisation a enlevé, a posteriori, toute légitimité à l'entreprise française outre-Méditerranée. Conquérir l'Algérie, l'occuper, y développer un peuplement européen, s'opposer pendant huit ans à son indépendance : autant d'actions dont la France ne peut s'enorgueillir.

La gestion de ce passé ne relève donc pas d'une gestion par deux États, anciens belligérants, qui se partageraient les torts des querelles passées, dans un mea culpa réciproque, ou tairaient leurs anciennes discordes pour fonder de nouvelles relations, comme dans le cas de la France et de l'Allemagne. Le rapport de domination coloniale a marqué et marque encore les relations entre la France et l'Algérie. Et les deux pays sont toujours, aujourd'hui, dans un rapport d'inégalité, la mondialisation ayant succédé à l'ère coloniale comme division du monde. La France est restée parmi les pays riches et puissants de la planète, tandis que l'Algérie n'est pas sortie du sous-développement. Les migrations entre les deux pays l'expriment parfaitement : alors que l'émigration est un besoin pour les Algériens, l'immigration est un "problème" pour le gouvernement français. C'est aux cris de "visa, visa " que le président Jacques Chirac a été accueilli en

1. "L'acculturation à la française signifiait d'un côté la possibilité de humer des vents frais de l'extérieur, voire d'en désirer la caresse. Le souffle de ces vents était employé à faire vaciller les étouffoirs communautaires du conservatisme algérien. D’un autre côté, cette acculturation était plus ou moins ressentie comme une trahison au regard des normes communautaires ", écrit Gilbert Meynier dans son Histoire intérieure du FLN, 1954-1962, op. cit., p. 47.
Algérie lors de sa visite officielle en mars 2003 et les Algériens installés en France vivent les mêmes difficultés que tous les immigrés en provenance du Maghreb ou des pays musulmans, avec l'émergence d'identités communautaires, dans une interrogation sur la définition de la nation française entre universalisme et communautarisme. C'est avec ces tensions que le passé est aujourd'hui regardé ${ }^{2}$. Une gestion commune ne supposerait-elle pas des relations plus équilibrées?

Enfin, la guerre civile actuelle en Algérie s'impose à la lecture du passé. Ce n'est pas le moindre des paradoxes que de voir des Algériens proches du pouvoir dénoncer la torture et la répression menées par la France entre 1954 et 1962 alors que, précisément, aujourd'hui, ils se taisent et même défendent le régime algérien, dont l'armée recourt à la torture, aux arrestations arbitraires et aux exécutions sommaires. Les disparitions de suspects ne sont pas, en Algérie, qu'une question passée ; elles sont aussi d'actualité.

L'incohérence apparente de cette position témoigne de la prégnance d'une logique nationaliste: dénoncer la torture pratiquée par la France entre 1954 et 1962, c'est légitimer, en regard, la construction de la nation algérienne ; taire aujourd'hui les pratiques des forces de l'ordre algériennes, c'est aussi défendre la nation algérienne. Pour ceux qui adoptent ce point de vue, les pratiques de répression n'ont pas la même valeur, ni le même sens, lorsqu'elles s'inscrivent dans un rapport de domination coloniale ou lorsqu'elles sont mises en œuvre dans un cadre national indépendant. C'est pourquoi les autorités algériennes mettent en avant l'ingérence insupportable que représenteraient les enquêtes menées par des commissions internationales pour les rejeter.

2. Significative de ce lien entre passé et présent, une émission de France 2, Mots Croisés, le 3 novembre 2003, a rassemblé deux débats : le premier sur le voile et la laïcité, le second sur le livre de Georges-Marc Benamou. 
Or, agiter, depuis l'Algérie, les crimes français du passé est une forme de réplique à la dénonciation, en France, des pratiques de répression du régime algérien actuel. À chacun ses crimes, à chacun ses fautes. Pour le mieux des relations francoalgériennes, il vaut mieux alors se taire. La protestation d'une soixantaine de parlementaires de la majorité contre la présence d'Abdelaziz Bouteflika aux cérémonies de commémoration du débarquement en Provence, le 15 août 2004, en témoigne : ils l'ont dénoncée au motif qu'il aurait " toujours bafoué "la mémoire des harkis ${ }^{1}$. La coopération actuelle, renouvelée, affichée entre les deux États, cependant, prime sur les litiges du passé. Leur gestion, en outre, s'inscrit dans une configuration complexe, liée à l'imbrication des camps en présence au moment de la guerre elle-même.

\section{O L'ABSENCE DE REPÈRES POUR GÉRER CE PASSÉ}

La politique française de reconnaissance et de réparation à l'égard des victimes du régime de Vichy n'offre guère de solution au cas franco-algérien. Les repères qui président à la gestion du passé douloureux de l'occupation allemande et de la collaboration sont brouillés dans le cas de la guerre d'indépendance algérienne. Où étaient les victimes? Où étaient les bourreaux? Qui est le vainqueur? Qui est le vaincu? Où étaient le Bien et le Mal ? Ces questions ouvrent une cascade d'interrogations, au lieu d'aboutir à des certitudes sur lesquelles pourrait se fonder une politique de gestion de ce passé.

Pendant la guerre d'indépendance algérienne, les victimes l'ont été de part et d'autre : outre les Algériens victimes de la répression des forces françaises et, en même temps, des violences du FLN ou de l'ALN, il s'agissait aussi d'Algériens et de Français, engagés dans les deux camps. Les harkis étaient ainsi des Algériens se

1. Cf. notamment Le Monde, du 10 août 2004. battant aux côtés des troupes françaises, tandis que des Français se sont engagés avec les nationalistes algériens, en combattant avec eux, comme, par exemple, certains membres du PCA (Parti communiste algérien), ou en constituant, en métropole, des réseaux de soutien ${ }^{2}$.

Laissés-pour-compte du processus de paix mis en ouvre par les accords d'Évian, des harkis ont été massacrés en Algérie et rejetés par les autorités françaises, réticentes à les accueillir et les protéger. Les Français soutenant le FLN ou l'ALN ont pu, eux, être arrêtés, parfois torturés, condamnés, y compris à la peine de mort, et même, dans un cas, exécuté ${ }^{3}$. Dans le camp des partisans de l'Algérie française, les membres de l'OAS (Organisation de l'armée secrète) ont eux aussi subi la torture. Les piedsnoirs, enfin, ont vécu une fin de guerre traumatisante, entre départ précipité pour la métropole et enlèvements, parfois torture et disparitions du fait d'Algériens, sans oublier les morts du 5 juillet 1962, le jour même de l'indépendance ${ }^{4}$.

Constater la multiplicité des victimes de la guerre ne signifie pas mettre les deux camps sur un pied d'égalité, en gommant la question de la légitimité de leurs combats respectifs ou celle de l'ampleur prise par leurs violences. Simplement, du point de vue de la victime, l'effet du traumatisme est le même : il produit de la souffrance et légitime une demande de réparation. Or, la réponse à cette demande provoque une mise en concurrence des victimes, dont les

2. Hervé Hamon et Patrick Rotman les ont rendus célèbres sous l'expression de " porteurs de valise ", dans leur ouvrage : Les porteurs de valise. De la résistance française à la guerre d'Algérie, Paris, Albin Michel, 1979 (pour la 1 ère édition).

3. Il s'agit de Fernand Iveton. Cf. Jean-Luc Einaudi, Pour l'exemple. L'affaire Iveton. Enquête, Paris, L'Harmattan, 1986. Les affaires Alleg et Audin ont révélé, à l'époque même, la pratique de la torture : Henri Alleg, La Question, Paris, Minuit, 1958, et Pierre Vidal-Naquet, L'affaire Audin, Paris, Minuit, 1958.

4. Fouad Soufi en livre un récit aussi précis que possible dans "Oran, 28 février 1962-5 juillet 1962. Deux événements pour l'histoire, deux événements pour la mémoire ", in La guerre d'Algérie au miroir des décolonisations françaises, Actes du colloque en l'honneur de Charles-Robert Ageron, Paris, SFHOM, 2000, p. 635-676. 
bourreaux viennent des deux camps: forces de l'ordre françaises - police, armée, forces spéciales anti-OAS...- et nationalistes algériens.

De fait, lorsque les victimes de cette guerre réclament une reconnaissance de leurs souffrances, elles réclament aussi la stigmatisation et la condamnation d'un des deux camps, celui qui les a faits souffrir. C'est ainsi que leur demande réactive le conflit passé et aboutit, in fine, à rejouer la guerre. Le choix de la reconnaissance d'une catégorie de victimes revient à trancher la question de la légitimité du combat des deux camps en présence, soit celui de la France pour le maintien de l'Algérie française, soit celui du FLN pour l'accession à l'indépendance. Pourtant, sur le plan strictement humain, individuel, toutes les souffrances ne se valent-elles pas et ne méritent-elles pas réparation? Et comment y parvenir quand celle-ci impose un choix politique entre les deux camps, ex-adversaires de la guerre? Dans le cas du régime de Vichy, le choix n'était plus à faire, car les victimes, même en concurrence, ont toutes souffert d'un seul et même camp, vaincu et décrié, sans défenseur, celui du nazisme et de ses alliés.

Le choix pourrait être facilité si les catégories morales s'appliquaient sans discussion, assimilant l'un des deux camps au Bien ou au Mal. Mais les pratiques de violence utilisées de part et d'autre, sans cesse opposées l'une à l'autre, rendent difficile une telle catégorisation. Et, s'il est à peu près admis que l'accession de l'Algérie à l'indépendance s'inscrivait logiquement dans le processus de décolonisation marquant l'histoire du monde après 1945, légitimant ainsi, politiquement, la lutte des nationalistes algériens, cela n'empêche pas des discussions interminables sur les méfaits ou les bienfaits de la colonisation, bloquant son inscription claire et irréversible dans la catégorie du Mal. C'est pour cette raison, d'ailleurs, que certains ont recours aux catégories juridiques, en réclamant la qualification de la colonisation comme crime contre l'humanité, unanimement reconnu comme la catégorie du Mal suprême ${ }^{1}$.

La victoire des nationalistes algériens elle-même produit une légitimation qui peut se discuter, dans la mesure où les vainqueurs et les vaincus transcendent les barrières nationales. En effet, s'il semble évident que la France a perdu et que l'Algérie, au contraire, a gagné la guerre, cette vision se complique très largement, en raison du caractère multiple de ce conflit, qui s'est doublé d'une guerre civile dans chacun des camps. Des Algériens, ceux qui s'étaient placés du côté des Français, ont ainsi été vaincus. Quant aux Français opposés à la guerre, anticolonialistes ou militants contre la torture, ils peuvent être rangés dans la catégorie des vainqueurs. La nébuleuse des organisations et des hommes luttant contre la guerre a, d'une certaine façon, remporté une victoire en 1962, l'issue de la guerre les confortant dans leurs positions. L'histoire leur a donné raison.

En outre, a posteriori, la victoire est discutée, car le présent suscite une relecture du passé. Au vu de la situation actuelle en Algérie, des voix s'élèvent parfois, sur place, pour regretter, à demi-mots, la présence française, ou, plus couramment, pour déplorer que la victoire de 1962 n'ait pas été transformée, sur le long terme, en une réussite réelle. Les Algériens peuvent avoir le sentiment d'être les éternels vaincus de l'histoire. Parfois aussi, le discours populaire rend la France responsable de cette situation, avec des arguments totalement contradictoires : soit, en abandonnant l'Algérie, en s'en dégageant rapidement, elle l'aurait laissée livrée à elle-même ; soit, au contraire, en dirigeant le pays dans les coulisses, par collusion

1. Suivant une démarche identique au Livre noir du communisme (Paris, Robert Laffont, 1997), le récent Livre noir $d u$ colonialisme, en témoigne (Marc Ferro (dir.), Paris, Robert Laffont, 2002). Plusieurs historiens spécialistes de la colonisation et de la décolonisation en ont fait la critique dans Afrique et Histoire, 1, septembre 2003, p. 245-267. 
avec les dirigeants algériens, elle aurait précipité le pays dans l'abîme où il se trouve.

Au contraire de la seconde guerre mondiale, enfin, la résurgence de la guerre d'indépendance algérienne produit un renversement étonnant des rôles de victime et de bourreau. Il est très frappant de voir des criminels présentés comme des victimes, en raison du traumatisme provoqué par les violences qu'ils ont commises. C'est le cas pour les anciens d'Algérie qui ont pratiqué la torture ou commis des exécutions sommaires ${ }^{1}$. Cet effacement de la frontière entre bourreaux et victimes est d'autant plus accentué que ces criminels ont aussi été des victimes directes de la guerre, témoins de l'horreur d'un attentat, de la mort d'un " camarade " en embuscade ou de sa mutilation par l'adversaire ${ }^{2}$. Le discours de l'armée, s'estimant déshonorée par le retour du débat sur la torture, joue d'ailleurs sur un renversement des rôles : les bourreaux sont les autres, ceux du FLN qui ont utilisé le terrorisme, l'assassinat, le massacre de populations réticentes, les purges internes etc.

Ce manque de repères alimente la demande sociale, très forte aujourd'hui, en France, d'une "vérité "sur la guerre d'indépendance algérienne, qui serait bloquée par la fermeture des archives. Et le dialogue de sourds se répète à l'infini, lorsque les historiens objectent vainement l'existence de leurs travaux, fondés sur le dépouillement d'une quantité parfois impressionnante d'archives, à toute nouvelle et récurrente exigence de "vérité. "Mais, en réalité, le terme ne serait-il pas mal choisi ? Plus que la "vérité ", les Français ne cher-

1. C'est l'orientation du documentaire de Patrick Rotman, L'ennemi intime, diffusé en mars 2002 par France 3, accompagné d'une publication aux Éditions du Seuil. Pour Raphaëlle Branche, la " question initiale " de ce film est bien : "Comment vivre avec un événement présenté comme traumatique (pratiquer la torture, exécuter un prisonnier)?", Vingtième Siècle, Revue d'bistoire, 77, janvier-mars 2002, p. 117.

2. Raphaëlle Branche insiste sur cette expérience dans le passage à l'acte de torture, dans La torture et l'armée pendant la guerre d'Algérie, Paris, Gallimard, 2001. cheraient-ils pas plutôt des " certitudes " sur la légitimité des camps en présence et des demandes de réparation, sur les torts des uns et des autres ? Or, plongés dans la complexité de la guerre d'indépendance algérienne, les historiens peinent à leur en livrer.

Car, finalement, en dehors d'un point de vue moral selon lequel toutes les victimes ont droit à réparation, tout est un choix politique. Au-delà des innombrables souffrances générées par ce conflit, c'est indéniablement au cour même de ses multiples épaisseurs, de ses différentes strates, entre chaque camp mais aussi à l'intérieur de chacun d'entre eux, que gît la difficulté de gérer ce passé, producteur de mémoires discordantes, concurrentes et revendicatrices. Quand elle s'inscrit dans un cadre national, la gestion de ce passé implique un choix, générateur de polémiques, entre les deux camps ${ }^{3}$. Elle est ainsi condamnée à reproduire le conflit. La voie de la coopération franco-algérienne dans l'examen de ce passé apparaît ainsi comme la seule pertinente. À l'image de la réconciliation francoallemande, qui a permis aux Français d'oublier l'occupant allemand pour se focaliser sur le régime de Vichy, un rapprochement franco-algérien libérerait la gestion de ce passé de la figure de l'ennemi extérieur pour en permettre, ensuite, un traitement interne.

Les relations bilatérales franco-algériennes s'inscrivent cependant dans une conjoncture défavorable à un éventuel rapprochement sur le terrain mémoriel. Sans recourir abusivement au parallèle franco-allemand, le décalage avec la situation de la France et de l'Allemagne renforce cette idée : la réconciliation franco-allemande a bénéficié de la construction de l'Union européenne, jusqu'à en devenir, d'ailleurs, l'axe fort. La construction politique de l'Europe a joué à

3. La critique de Guy Pervillé sur le caractère "unilatéral " de la "repentance "se fonde sur ce constat : La revendication algérienne de repentance unilatérale, à paraître dans Némésis, 5, 2004. 
la fois sur ceux qui étaient favorables à la réconciliation franco-allemande et sur ceux qui y étaient réticents : pour les premiers, elle s'est présentée comme une opportunité à saisir tandis que, envers les seconds, elle est apparue comme une contrainte. L'enjeu de la réconciliation franco-allemande n'était rien moins que la construction de l'Europe en tant qu'entité politique. Les rapports entre la France et l'Algérie, eux, ont été et restent des rapports Nord-Sud, sans perspective de politique internationale favorable. La commémoration franco-algérienne, en outre, n'a donc pas trouvé de moment opportun, ni d'hommes d'État susceptibles de la mener.

L'analyse de la gestion du passé de la guerre d'indépendance algérienne par les deux pays ramène ainsi au présent, qui " commande au passé ${ }^{1}$ ". Elle met en jeu la nature des régimes, la conjoncture actuelle, interne, des deux pays, ainsi que le contexte international. À l'heure d'une coopération franco-algérienne, pourtant, la France et l'Algérie ne pourraient-elles pas chercher à appréhender ensemble, conjointement, cette histoire?

Chargée de recherche à l'Institut d'bistoire $d u$ temps présent (IHTP), Sylvie Thénault (thenault@ihtp.cnrs.fr), auteure d'Une drôle de Justice. Les magistrats pendant la guerre d'Algérie (La Découverte, 2004 [rééd.]) est spécialiste des questions de répression légale des nationalistes algériens, par la justice et l'internement.

1. Comme l'écrit Benjamin Stora, dans "Maroc-Algérie. Retour du passé et écriture de l'histoire ", op. cit., p. 109. 